\title{
Reversible Sliding in Networks of Nanowires
}

\author{
Jianghong Wu, ${ }^{\dagger, \neq, \|}$ Jianfeng Zang, ${ }^{\S, \|}$ Aaron R. Rathmell, ${ }^{\dagger}$ Xuanhe Zhao, ${ }^{*}{ }^{\S}$ and Benjamin J. Wiley ${ }^{*}{ }^{\dagger}$ \\ ${ }^{\dagger}$ Department of Chemistry, Duke University, 124 Science Drive, Box 90354, Durham, North Carolina 27708, United States \\ ${ }^{*}$ Department of Materials Science and Engineering, Donghua University, Shanghai 201620, China \\ ${ }^{\S}$ Soft Active Materials Laboratory, Department of Mechanical Engineering and Materials Science, Duke University, Durham, \\ North Carolina 27708, United States
}

\section{Supporting Information}

ABSTRACT: This work demonstrates that metal nanowires in a percolating network can reversibly slide across one another. Reversible sliding allows networks of metal nanowires to maintain electrical contact while being stretched to strains greater than the fracture strain for individual nanowires. This phenomenon was demonstrated by using networks of nanowires as compliant electrodes for a dielectric elastomer actuator. Reversible nanowire sliding enabled actuation to a maximum area strain of $200 \%$ and repetitive cycling of the actuator to an area strain of $25 \%$ over 150 times. During actuation, the transmittance of the network increased 4.5 times, from $13 \%$ to $58 \%$. Compared to carbon-based compliant electrodes, networks of metal nanowires can actuate across a broader range of optical transmittance. The widely tunable transmittance of nanowire-based actuators allows for their use as a light valve.

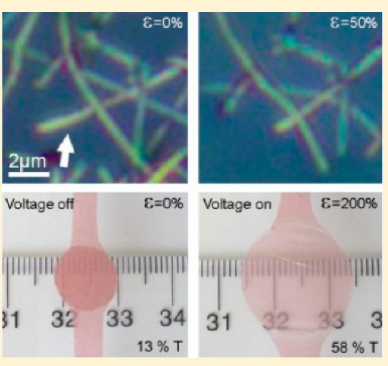

KEYWORDS: Copper nanowires, transparent electrode, actuator, sliding, light valve

$\mathrm{T}$ he discovery of new ways to reversibly deform materials to strains greater than their intrinsic limits without irreversibly changing their electrical properties remains of fundamental importance to the development of stretchable displays, ${ }^{1-4}$ skinlike sensors of pressure, ${ }^{5,6}$ haptic feedback, ${ }^{7}$ harvesters of mechanical energy, ${ }^{8}$ electronic textiles, ${ }^{9}$ responsive surfaces and interfaces, ${ }^{10}$ and muscle-like soft actuators. ${ }^{11-13}$ Here we report that metal nanowires in a large-area network can reversibly slide across one another, enabling repeated deformation of the network while retaining electrical conductivity. We demonstrate the use of sliding networks of nanowires as compliant electrodes for dielectric elastomer actuators. The network of sliding nanowires could be actuated to a maximum area strain of $225 \%$ and a transmittance of $76 \%$. Reversible nanowire sliding enabled repetitive cycling of the actuator to a strain of $25 \%$ over 150 times. The widely tunable transmittance of nanowire-based actuators further allows for their use as a light valve.

The dominant approach to avoid failures of conductive components upon stretching is by making them wavy or buckled in shape.,14-19 For example, buckled ribbons of silicon can exhibit strains of $20 \%$, which is 20 times greater than the intrinsic fracture limit of bulk silicon. ${ }^{17}$ Connecting buckled structures to nodes in a mesh architecture allowed for the fabrication of a hemispherical camera, ${ }^{18}$ a stretchable LED display, ${ }^{3}$ and silicon-based circuits that can be stretched up to $140 \%{ }^{19}$ The manufacturing of these and other stretchable mesh architectures with buckled, spring-like, or serpentine interconnects requires photolithographic patterning and high-fidelity transfer-printing steps that limits their utility to small-area applications that are relatively insensitive to fabrication cost.

Networks of one-dimensional nanomaterials have emerged as an alternative approach to obtain highly stretchable conductive materials with simple, scalable processing steps (e.g., solutionphase mixing and coating). ${ }^{20-22}$ For the case of stretchable carbon nanotube films and composites, both the deformation of the network and buckling of nanotubes can play a role in maintaining conductivity at high strains. ${ }^{1,5,12,23,24}$ The stretchability of carbon nanotube networks has been utilized in the construction of a stretchable active matrix display, ${ }^{1}$ a stretchable OLED, ${ }^{25}$ a skin-like sensor of pressure and strain, ${ }^{5}$ and a faulttolerant compliant electrode for an electrostatic actuator that can achieve an area strain of $200 \%{ }^{12}$ Silver nanowires embedded in a polymer matrix have also recently been used to make a compliant electrode, but it was actuated to an area strain of only $68 \% .^{26}$ In addition, the mechanisms enabling the stretchability of one-dimensional nanowire networks without prebuckling have not yet been revealed.

Here we demonstrate that metal nanowires can reversibly slide across one another while retaining electrical contact. Metal nanowires undergo plastic deformation and fracture at strains of $1-5 \%,{ }^{27}$ but sliding allowed for networks of nanowires to be used as compliant electrodes for electrostatic actuators with maximum area strains of $200 \%$ - triple that of previous results utilizing metal nanowires. ${ }^{26}$ Sliding further enables the transmittance of the metal nanowire networks to be tuned over a range 3 times that of carbon nanotube networks. Finally, we show the maximum strain and transmittance of the nanowire networks can be understood in terms of the critical number of nanowires per unit area necessary for percolation.

Received: January 7, 2013

Revised: April 8, 2013

Published: May 1, 2013 

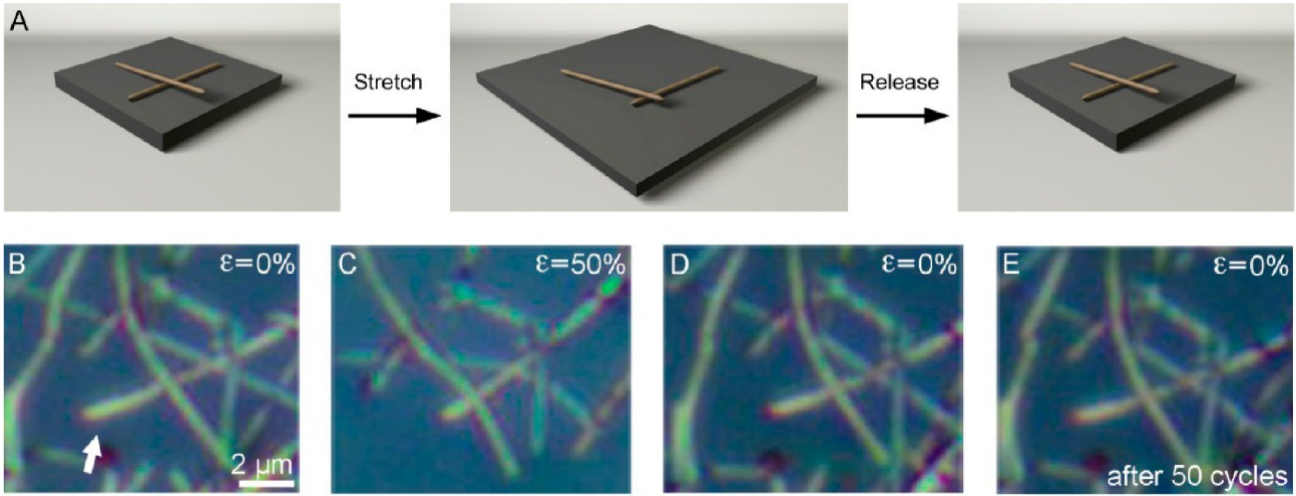

Figure 1. (A) Schematic illustration of nanowires sliding on an elastomeric substrate. (B, C) Sequential pictures of a nanowire sliding as the strain is varied from $0 \%$ to $50 \%$. (D, E) The nanowire returns to its starting position as the strain is relaxed back to $0 \%$, even after 50 cycles of actuation.
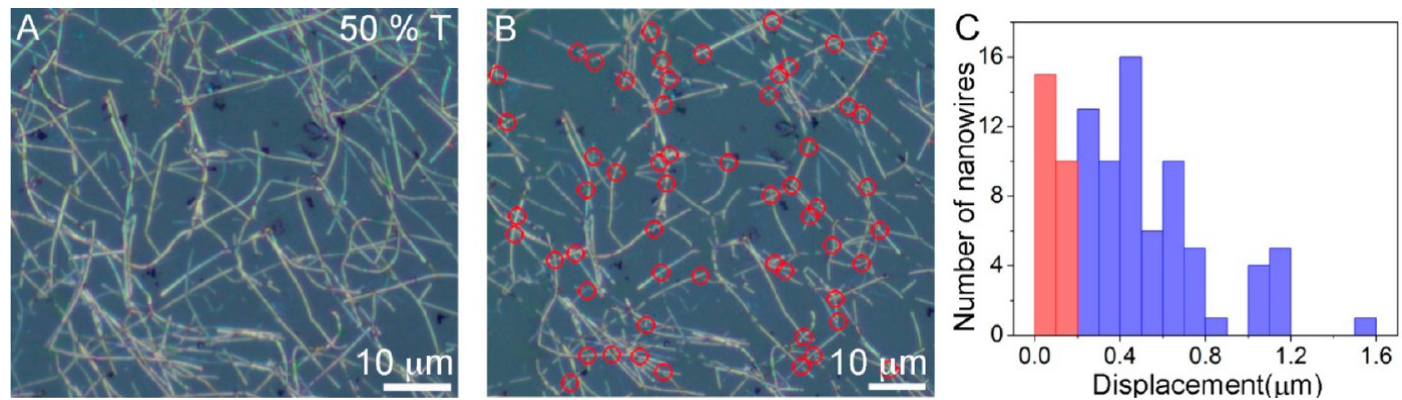

Figure 2. Microscope images of a copper nanowire network at an area strain of (A) $0 \%$ and (B) $20 \%$. Red circles in panel B indicate contact points between nanowires. (C) Distribution of the displacement of nanowires at the contact points in panel B. The red bars indicate displacements that are considered to be within the error of measurement $(\leq 0.2 \mu \mathrm{m})$, which represent $25 \%$ of all contact points.

We observed the deformation of a network of copper nanowires on an elastomeric substrate (3M VHB 4905 acrylic elastomer, VHB) under an applied strain using an optical microscope (Figure 1). As indicated by the schematic in Figure $1 \mathrm{~A}$, the nanowires are laying on the polymer surface and are not embedded in polymer. Figures $1 \mathrm{~B}, \mathrm{C}$ show one nanowire sliding across another as the substrate is stretched to a strain of $50 \%$, moving $2 \mu \mathrm{m}$ from its initial position. As the strain is decreased back to $0 \%$, the nanowire returns to its starting position (Figure 1D). Even after 50 cycles of stretching to a strain of $50 \%$, the nanowire returns to roughly the same starting position (Figure $1 \mathrm{E}$ ). Figure S1 shows microscope images of three additional examples of nanowire sliding. To better quantify the extent of nanowire sliding, the displacement of nanowires from contact points was measured before and after stretching a nanowire network with a transmittance of $50 \%(\lambda=550 \mathrm{~nm})$ to an area strain of $20 \%$. Figure 2 illustrates that nanowire displacement greater than $0.2 \mu \mathrm{m}$ occurred at $75 \%$ of all contact points. To our knowledge, this is the first confirmation that nanowires in a network can reversibly slide across one another.

In order to examine the properties of a network of sliding nanowires, we tested their performance as the top and bottom electrodes of a dielectric elastomer actuator (Figure 3A). To make this device, copper nanowires were filtered from solution onto porous membranes, the membranes with the copper nanowires were cut into the desired shape, and the nanowire films were transferred from the membranes to a prestretched (to an area strain of $300 \%$ ) VHB film by putting them into contact and pressing by hand (Figure S2 provides a visual illustration of this fabrication process). As with previous studies that utilized carbon nanotubes for the dielectric elastomer actuator's electrodes ${ }^{16}$ the nanowires on the VHB surface were not embedded in an additional polymer overcoat. To induce actuation, a direct-current voltage was applied to the top and bottom copper nanowire electrodes. We note that the diameters $(10 \mathrm{~mm})$ of the electrodes are much larger than the thickness $(30 \mu \mathrm{m})$ of the dielectric elastomer film, and the average mesh size of the nanowire network $(<5 \mu \mathrm{m})$ is smaller than the film thickness. Therefore, we can approximate the mesh of nanowires as a continuous electrode, with edge effects having a negligible impact on the deformation of the actuator.

Figures 3B,C demonstrate that the copper nanowire electrodes can achieve an area strain greater than $200 \%$ at an applied voltage of $4.8 \mathrm{kV}$ for a $30 \mu \mathrm{m}$ thick VHB film. This value is comparable to the highest previous area strain ( 200\%) obtained using carbon nanotubes. ${ }^{12}$ During actuation, the transmittance of the actuator increased 4.5 times from $13 \%$ to $58 \%$. In comparison, the transmittance of carbon nanotube electrodes could only be increased by a factor of 1.4. ${ }^{7}$ Figures 3D,E demonstrate significant actuation can also be achieved at a relatively high transmittance. This film, with an initial transmittance of $65 \%$, increased to a transmittance of $76 \%$ as it was actuated to an area strain of $54 \%$. We note that these transmittance values are at a wavelength of $550 \mathrm{~nm}$. For reference, the full broadband optical transmittance spectra of the nanowire actuators are given in Figure S3.

Figures 3F,G show how the nanowire network evolves during actuation. The nanowire network starts in a state in which nearly every nanowire is in contact with at least two other nanowires. We denote the number of nanowires per unit area at the initial state as $N_{0}$. As a voltage is applied and the area of the network increases, the number of nanowires per unit area at the 

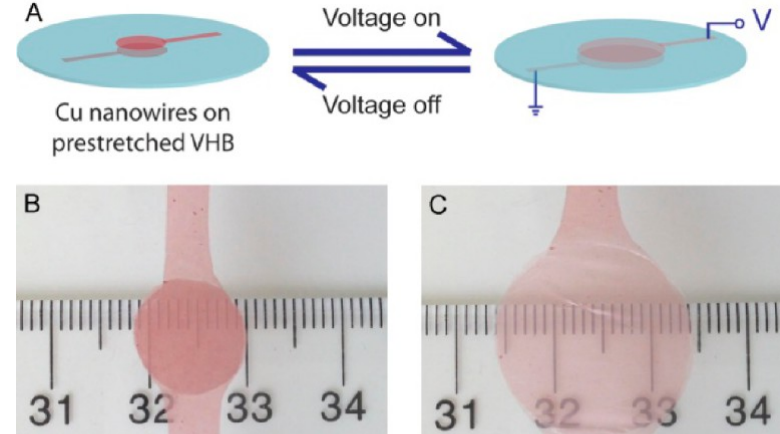

$13 \% \mathrm{~T}$
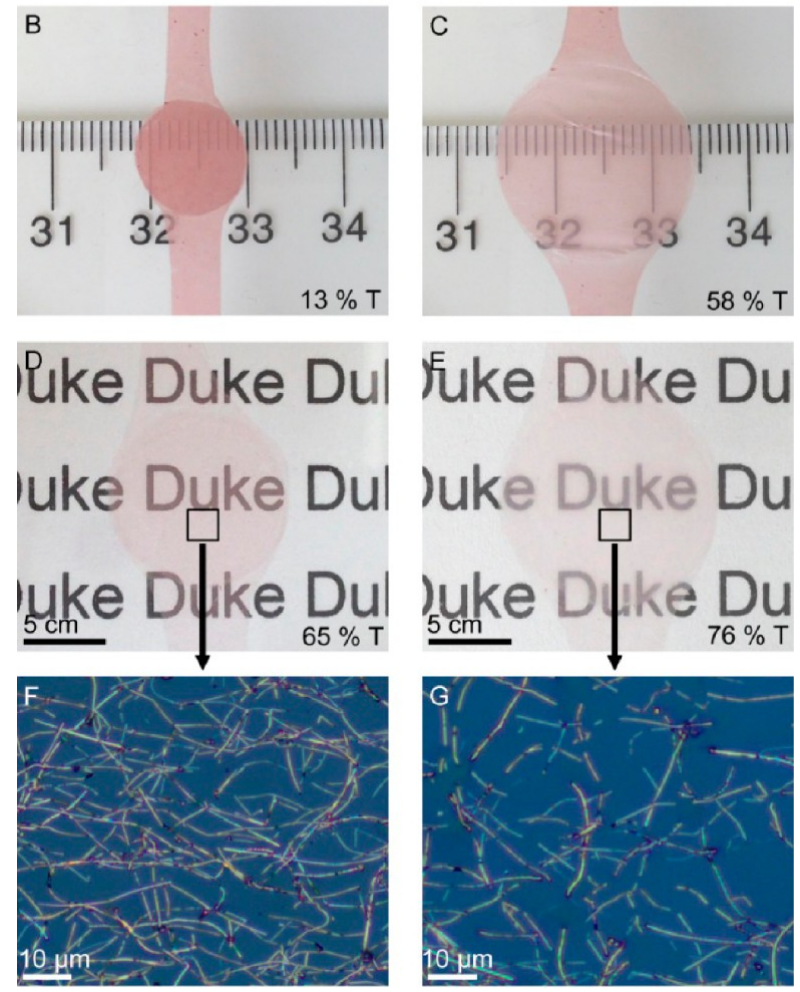

Figure 3. (A) Schematic of a transparent copper nanowire actuator. (B, C) An actuator with a transmittance of $13 \%$ at rest increases to a transmittance of $58 \%$ as it is actuated to an area strain of $200 \%$ with an applied voltage of $4.8 \mathrm{kV}$. (D, E) An actuator with a starting transmittance of $65 \%$ can be actuated to a transmittance of $76 \%$ at an area strain of $54 \%$ and a voltage of $4.8 \mathrm{kV}$. (F, G) Microscope images showing the networks of copper nanowires on VHB in (D) and (E), respectively.

current state $N$ will decrease following $N=N_{0}(1+\varepsilon)^{-1}$, where $\varepsilon$ is the area strain of actuation. Once $N$ approaches a critical area density of nanowires required for electrical percolation $N_{\mathcal{c}}$ the nanowire network ceases to be conductive. According to percolation theory, the critical area density of nanowires is given by $N_{\mathrm{c}}=5.71 \mathrm{~L}^{-2}$, where $L$ is the length of the nanowires. ${ }^{28}$ For the copper nanowires used here, $L=38 \pm 11.2 \mu \mathrm{m}$, and thus $N_{\mathrm{c}}$ is approximately $4 \times 10^{9} \mathrm{~m}^{-2}$.

Figures $4 A, B$ illustrate the relationship between the initial area density of nanowires, the area strain, and the transmittance of the nanowire networks. For a network of nanowires with an initial area density of $8 N_{c}$ and a transmittance of $64 \%$, the maximum area strain was $50 \%$, to give a final area density of $5 N_{c}$. A network of nanowires with an initial area density of $38 N_{c}$ and a transmittance of $10 \%$ can be electrically actuated to 3 times its original size, to give a final area density of $11 N_{c}$ and a transmittance of $54 \%$.

We observed that for nanowire networks with low initial area densities (i.e., $8-16 N_{c}$ ) the maximum area strain occurred at a number density of $5-7 N_{\mathrm{c}}$. Given that nanowire networks have been shown to be fairly conductive $\left(\sim 500 \Omega \mathrm{sq}^{-1}\right)$ at $2 \mathrm{~N}_{\mathrm{c}}{ }^{29}$ this limit is not likely due to a drop in conductivity. Rather, the areas of unconnected nanowires visible at a number density of
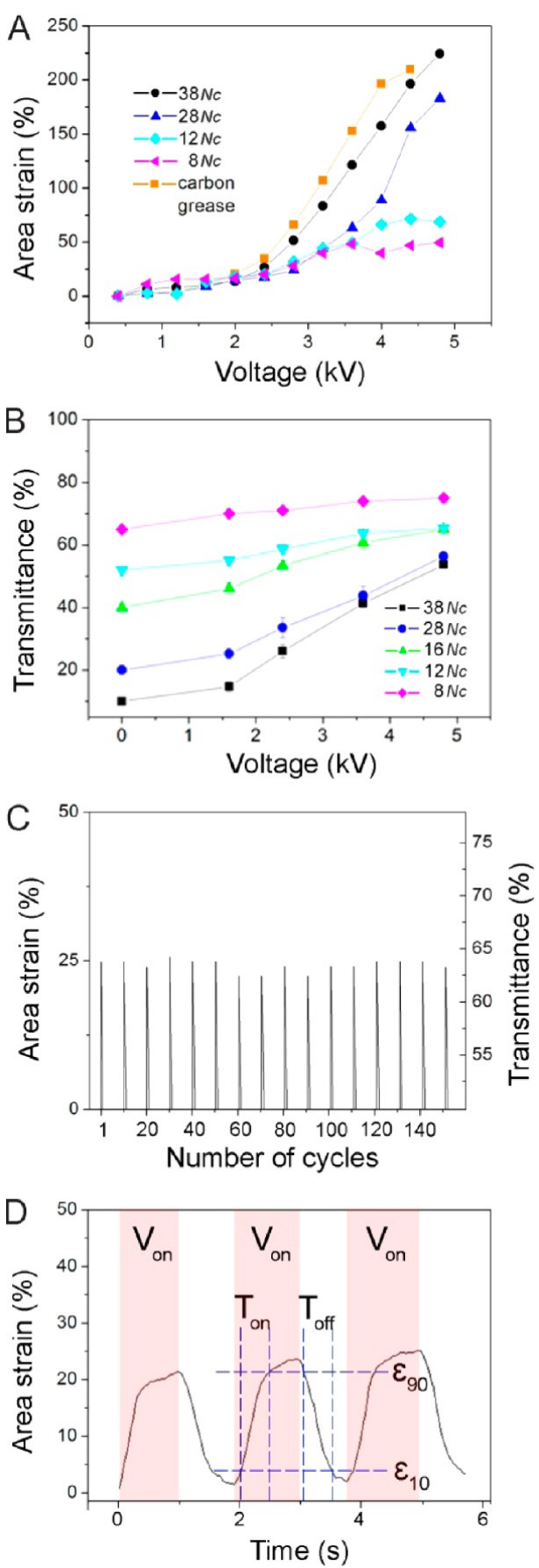

Figure 4. (A) Voltage-induced actuation of copper nanowire actuators at different number densities compared to a conventional carbon grease electrode. Here $N_{c}$ is the critical density required for percolation, which in this case is $4 \times 10^{9}$ nanowires $\mathrm{m}^{-2}$. (B) Transmittance $(\lambda=550 \mathrm{~nm})$ of copper nanowire actuators at different densities as a function of applied voltage. (C) Area strain actuation and transmittance variation as a function of applied voltage during 150 continuous cycling tests. The number density of copper nanowires is $12 N_{\mathcal{c}}$ the applied voltage is $3.2 \mathrm{kV}$, and the data were collected every 10 cycles. (D) Illustration of how strain varied with time for three consecutive cycles of actuation.

$5 N_{c}$ (Figure 3G) indicate the network begins to lose its connectivity at this number density. This lack of connectivity will likely limit the charge density that can be achieved on the film and thus limit the maximum area strain. However, this qualitative explanation remains a hypothesis, and additional 

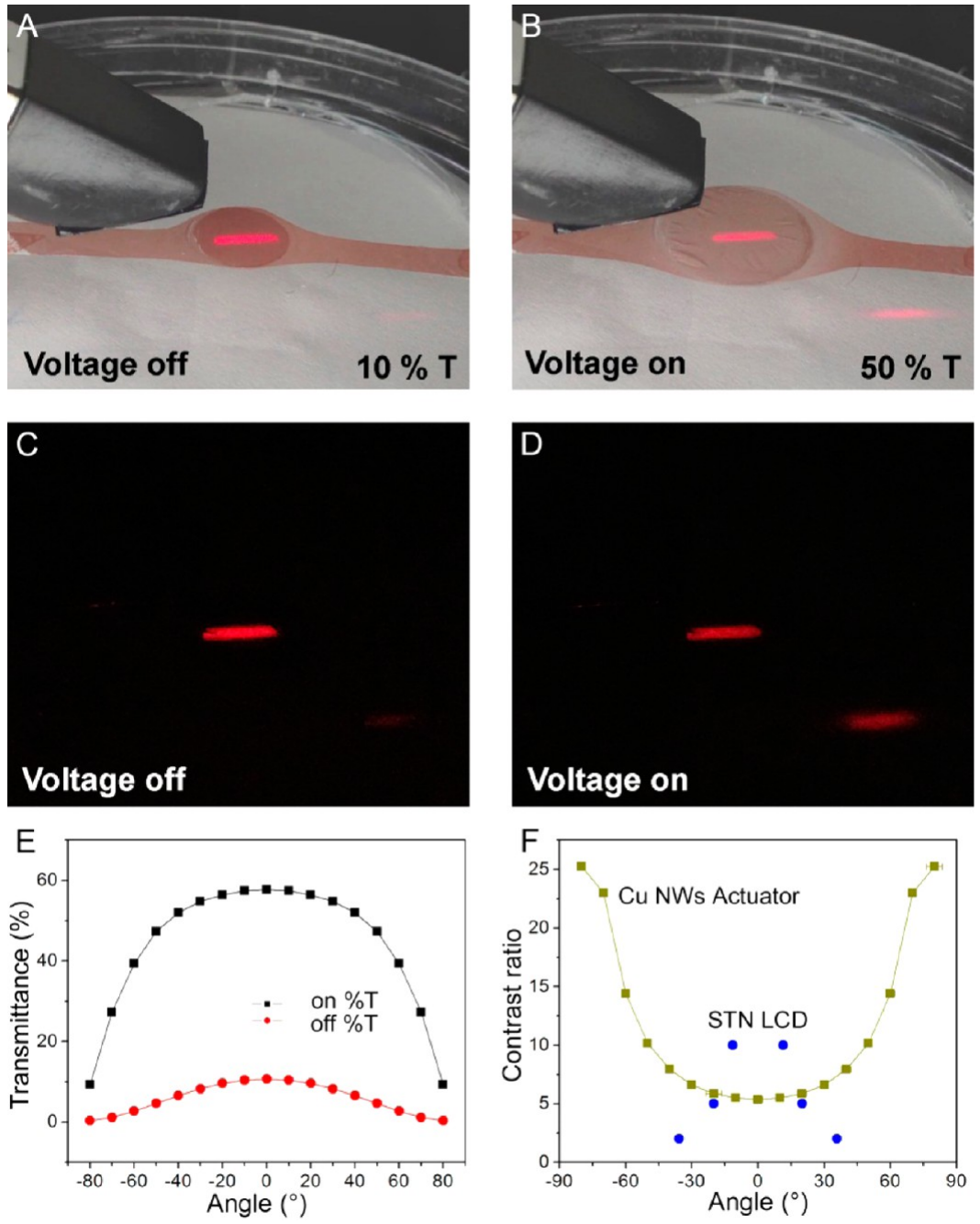

Figure 5. Pictures of a copper nanowire actuator serving as a light valve for a laser with the room lights on (A, B) and off (C, D). (E) Transmittance of the nanowire light valve as a function of incident angle (relative to normal) for both the on and off states. (F) Contrast ratio as a function of angle for the nanowire light valve and an uncompensated supertwisted nematic liquid crystal displays (STN LCD).

theoretical simulations are necessary to quantify the degree of connectivity at a given number density of nanowires. In contrast, nanowire networks with higher initial area densities (i.e., $28-38 N_{c}$ ) could be actuated to a number density no lower than $10-11 N_{\mathcal{c}}$ and nanowire networks with initial area densities greater than $38 N_{c}$ had lower maximum actuation strains. This difference is likely due to the fact that higher initial area densities of nanowires led to nanowire entanglement, which hindered sliding. As shown in Figure S4, nanowire entanglement at high nanowire densities led to nanowire fracture and clumps of nanowires (Figure S4B) that were not apparent at low nanowire densities (Figure S4E). Figures S4C and S4F show the length distribution before and after actuation to the maximum area strain for Figures 4A,B and 4D,E, respectively. The histograms indicate that high nanowire densities led to greater fracture of nanowires than low nanowire densities. Initially, the average length of the nanowires was $25 \pm 7.7 \mu \mathrm{m}$, but this decreased to $4 \pm 2.4 \mu \mathrm{m}$ at a density of $11 N_{c}$ and $13 \pm 4.7 \mu \mathrm{m}$ at a density of $7 N_{c}$.

To demonstrate the generality of the sliding mechanism, we also examined the use of silver nanowires as compliant electrodes for actuators (Figure S5). For silver nanowires used in the current study, $L=14 \pm 5.3 \mu \mathrm{m}$, which gives $N_{\mathrm{c}}=2.7 \times 10^{10} \mathrm{~m}^{-2}$. For a silver nanowire electrode with $N_{0}=17 N_{\mathrm{c}}$ and an initial transmittance of $21 \%$, the maximum actuation strain was $160 \%$. This gives a minimum number density of $7 N_{\mathcal{c}}$ which is within the range observed for copper nanowire networks. In addition, copper nanowire electrodes with the same initial transmittance achieved a maximum strain of $180 \%$ under the same conditions. Therefore, it appears the performance of copper and silver nanowire networks as compliant electrodes are roughly comparable due to the same sliding mechanism (see Tables S1 and S2 for a quantitative summary of the relationship between transmittance, area density, and actuation strain). However, copper nanowires may be more attractive for this and related applications due to their lower cost. ${ }^{30}$ Copper nanowires can also be coated with nickel to be made 100 times more resistance to oxidation than silver nanowires. ${ }^{31}$

Reliable and fast operation is critical to practical applications of dielectric elastomer actuators. Figure 4C presents the results of cycling an actuator with an initial transmittance of $50 \%\left(N_{0}=\right.$ $12 N_{c}$ ) between a strain of 0 and $25 \%$. The actuator was able to deliver area strains of $25 \pm 0.9 \%$ at a transmittance of $62 \pm$ $2.2 \%$ over 150 cycles, demonstrating the electrode's capability of repeated deformation to moderate strains. Figure 4D further shows that it took $0.5 \mathrm{~s}$ for the actuator to increase from an area strain of $2.2 \%$ to $20 \%$ under an applied voltage and $0.4 \mathrm{~s}$ for the actuator to relax once the voltage was withdrawn. This corresponds 
to a rate of actuation of $32 \% \mathrm{~s}^{-1}$ for increasing strain and $36 \% \mathrm{~s}^{-1}$ for decreasing strain. This response rate is comparable to that of VHB films actuated with carbon grease electrodes. ${ }^{32}$

The ability to electrically tune the transmittance of the actuators over a range of up to $44 \%$ represents a new opportunity to explore the use of nanowire networks as a light valve. In comparison to mechanical and electrochromic shutters, which typically operate between on/off states, copper nanowire-based light valves can offer intermediate levels of opacity. Compared to liquid-crystal light valves, copper nanowire-based light valves can offer a higher maximum transmittance (the maximum transmittance of liquid crystal light valve is $45 \%$ ), the ability to stretch and conform to different substrates, and much simpler construction. Figures 5A-D illustrate the capability of the copper nanowire actuator to act as a light valve. The laser is at an incident angle of $60^{\circ}$ from normal to illustrate the relatively large range of angles across which the nanowire actuator can act as a light valve. The full angle-dependent transmittance and contrast ratio of the copper nanowire actuator is given in Figures 5E,F. The copper nanowire actuator has a broader angular range over which it can act as a light valve compared to an uncompensated supertwisted nematic liquid-crystal display. ${ }^{33}$ This is due to the fact that, rather than using polarization-based optics to modify transmittance, the copper nanowire actuator is essentially a nanoscale version of a mechanical shutter.

In summary, networks of sliding nanowires represent a new way to achieve large mechanical strains for soft actuators in biomimetic robots, prosthetics, loudspeakers, tunable optics, and tactile (Braille) interfaces. ${ }^{34}$ Compared to carbon-based compliant electrodes, networks of metal nanowires can actuate across a broader range of transparent states and thus also serve as a valve for light. As the maximum strain that can be achieved for sliding nanowire networks is directly related to the critical area density required for percolation, the use of longer nanowires could allow for even greater ranges of actuation and transmittance. Finding a way to allow dense networks of nanowires to disentangle without fracture will also likely lead to increased maximum strains and improved contrast ratios.

\section{ASSOCIATED CONTENT}

\section{S Supporting Information}

Additional figures and tables. This material is available free of charge via the Internet at http://pubs.acs.org.

\section{AUTHOR INFORMATION}

\section{Corresponding Author}

*E-mail: benjamin.wiley@duke.edu (B.J.W.); xz69@duke.edu (X.Z.).

\section{Author Contributions}

"J.W. and J.Z. contributed equally to this work.

\section{Notes}

The authors declare no competing financial interest.

\section{ACKNOWLEDGMENTS}

B.J.W. and X.Z. acknowledge startup funding from Duke University and NSF's Research Triangle MRSEC (DMR1121107). J.W was supported by a fellowship from the China Scholarship Council. A.R.R. was supported by a Paul M. Gross Fellowship from the Department of Chemistry at Duke University and a fellowship from the NSF's Research Triangle MRSEC. X.Z. acknowledges the support from NSF (CMMI-
1253495 and CMMI-1200515). The authors also thank Dr. Shengrong Ye for his assistance with the image processing.

\section{REFERENCES}

(1) Sekitani, T.; Noguchi, Y.; Hata, K.; Fukushima, T.; Aida, T.; Someya, T. Science 2008, 321 (5895), 1468-1472.

(2) Rogers, J. A.; Someya, T.; Huang, Y. G. Science 2010, 327 (5973), 1603-1607.

(3) Park, S. I.; Xiong, Y. J.; Kim, R. H.; Elvikis, P.; Meitl, M.; Kim, D. H.; Wu, J.; Yoon, J.; Yu, C. J.; Liu, Z. J.; Huang, Y. G.; Hwang, K.; Ferreira, P.; Li, X. L.; Choquette, K.; Rogers, J. A. Science 2009, 325 (5943), 977-981.

(4) Sekitani, T.; Nakajima, H.; Maeda, H.; Fukushima, T.; Aida, T.; Hata, K.; Someya, T. Nat. Mater. 2009, 8 (6), 494-499.

(5) Lipomi, D. J.; Vosgueritchian, M.; Tee, B. C. K.; Hellstrom, S. L.; Lee, J. A.; Fox, C. H.; Bao, Z. Nat. Nanotechnol. 2011, 6 (12), 788792.

(6) Someya, T.; Kato, Y.; Sekitani, T.; Iba, S.; Noguchi, Y.; Murase, Y.; Kawaguchi, H.; Sakurai, T. Proc. Natl. Acad. Sci. U. S. A. 2005, 102 (35), 12321-12325.

(7) Yu, Z.; Yuan, W.; Brochu, P.; Chen, B.; Liu, Z.; Pei, Q. Appl. Phys. Lett. 2009, 95 (19), 192904.

(8) Donelan, J. M.; Li, Q.; Naing, V.; Hoffer, J. A.; Weber, D. J.; Kuo, A. D. Science 2008, 319 (5864), 807-810.

(9) Service, R. F. Science 2003, 301 (5635), 909-911.

(10) Wang, Q.; Tahir, M.; Zang, J.; Zhao, X. Adv. Mater. 2012, 24 (15), 1947-1951

(11) Aliev, A. E.; Oh, J.; Kozlov, M. E.; Kuznetsov, A. A.; Fang, S.; Fonseca, A. F.; Ovalle, R.; Lima, M. D.; Haque, M. H.; Gartstein, Y. N.; Zhang, M.; Zakhidov, A. A.; Baughman, R. H. Science 2009, 323 (5921), 1575-1578.

(12) Yuan, W.; Hu, L.; Yu, Z.; Lam, T.; Biggs, J.; Ha, S. M.; Xi, D.; Chen, B.; Senesky, M. K.; Gruner, G.; Pei, Q. Adv. Mater. 2008, 20 (3), 621-625.

(13) Zang, J. F.; Ryu, S.; Pugno, N.; Wang, Q. M.; Tu, Q.; Buehler, M. J.; Zhao, X. H. Nat. Mater. 2013, 12, 321-325.

(14) Zhu, Y.; Xu, F. Adv. Mater. 2012, 24 (8), 1073-1077.

(15) Xu, F.; Wang, X.; Zhu, Y. T.; Zhu, Y. Adv. Funct. Mater. 2012, 22 (6), 1279-1283.

(16) Zhang, Y. Y.; Sheehan, C. J.; Zhai, J. Y.; Zou, G. F.; Luo, H. M.; Xiong, J.; Zhu, Y. T.; Jia, Q. X. Adv. Mater. 2010, 22 (28), 3027-3031.

(17) Jiang, H.; Khang, D.-Y.; Song, J.; Sun, Y.; Huang, Y.; Rogers, J. A. Proc. Natl. Acad. Sci. U. S. A. 2007, 104 (40), 15607-15612.

(18) Ko, H. C.; Stoykovich, M. P.; Song, J.; Malyarchuk, V.; Choi, W. M.; Yu, C.-J.; Geddes, J. B., III; Xiao, J.; Wang, S.; Huang, Y.; Rogers, J. A. Nature 2008, 454 (7205), 748-753.

(19) Kim, D.-H.; Song, J.; Choi, W. M.; Kim, H.-S.; Kim, R.-H.; Liu, Z.; Huang, Y. Y.; Hwang, K.-C.; Zhang, Y.-w.; Rogers, J. A. Proc. Natl. Acad. Sci. U. S. A. 2008, 105 (48), 18675-18680.

(20) Shang, Y. Y.; He, X. D.; Li, Y. B.; Zhang, L. H.; Li, Z.; Ji, C. Y.; Shi, E. Z.; Li, P. X.; Zhu, K.; Peng, Q. Y.; Wang, C.; Zhang, X. J.; Wang, R. G.; Wei, J. Q.; Wang, K. L.; Zhu, H. W.; Wu, D. H.; Cao, A. Y. Adv. Mater. 2012, 24 (21), 2896-2900.

(21) Gu, G.; Schmid, M.; Chiu, P. W.; Minett, A.; Fraysse, J.; Kim, G. T.; Roth, S.; Kozlov, M.; Munoz, E.; Baughman, R. H. Nat. Mater. 2003, 2 (5), 316-319.

(22) Lee, P.; Lee, J.; Lee, H.; Yeo, J.; Hong, S.; Nam, K. H.; Lee, D.; Lee, S. S.; Ko, S. H. Adv. Mater. 2012, 24 (25), 3326-3332.

(23) Yu, Z.; Niu, X.; Liu, Z.; Pei, Q. Adv. Mater. 2011, 23 (34), 3989-3994.

(24) Hu, L.; Yuan, W.; Brochu, P.; Gruner, G.; Pei, Q. Appl. Phys. Lett. 2009, 94 (16), 161108.

(25) Yu, Z.; Zhang, Q.; Li, L.; Chen, Q.; Niu, X.; Liu, J.; Pei, Q. Adv. Mater. 2011, 23 (5), 664-668.

(26) Yun, S.; Niu, X.; Yu, Z.; Hu, W.; Brochu, P.; Pei, Q. Adv. Mater. 2012, 24 (10), 1321-1327.

(27) Zhu, Y.; Qin, Q.; Xu, F.; Fan, F.; Ding, Y.; Zhang, T.; Wiley, B. J.; Wang, Z. L. Phys. Rev. B 2012, 85 (4), 045443.

(28) Pike, G. E.; Seager, C. H. Phys. Rev. B 1974, 10 (4), 1421-1434. 
(29) Bergin, S. M.; Chen, Y. H.; Rathmell, A. R.; Charbonneau, P.; Li, Z. Y.; Wiley, B. J. Nanoscale 2012, 4 (6), 1996-2004.

(30) Rathmell, A. R.; Bergin, S. M.; Hua, Y. L.; Li, Z. Y.; Wiley, B. J. Adv. Mater. 2010, 22 (32), 3558-3563.

(31) Rathmell, A. R.; Nguyen, M.; Chi, M. F.; Wiley, B. J. Nano Lett. 2012, 12 (6), 3193-3199.

(32) Pelrine, R.; Kornbluh, R.; Pei, Q. B.; Joseph, J. Science 2000, 287 (5454), 836-839.

(33) Scheffer, T.; Nehring, J. Annu. Rev. Mater. Sci. 1997, 27, 555583.

(34) Brochu, P.; Pei, Q. B. Macromol. Rapid Commun. 2010, 31 (1), $10-36$. 